\title{
Lateral mass screw stimulation thresholds in posterior cervical instrumentation surgery: a predictor of medial deviation
}

\author{
*Bayard Wilson, BA, ${ }^{1}$ Erik Curtis, MD, ${ }^{2}$ Brian Hirshman, MD, MS, ${ }^{2}$ Ahmet Oygar, MD, ${ }^{2}$ \\ Karen Chen, MD, ${ }^{3}$ Brandon C. Gabel, MD, ${ }^{2}$ Florin Vaida, PhD, ${ }^{4}$ David W. Allison, PhD, ${ }^{5}$ and \\ Joseph D. Ciacci, MD²
}

${ }^{1}$ School of Medicine, ${ }^{2}$ Department of Neurosurgery, ${ }^{3}$ Department of Radiology, ${ }^{4}$ Department of Family Medicine and Public Health, Division of Biostatistics and Bio-informatics, and ${ }^{5}$ Department of Interventional Neurophysiology, University of California, San Diego, California

\begin{abstract}
OBJECTIVE Normative data exists for stimulus-evoked pedicle screw electromyography (EMG) current thresholds in the lumbar spine, and is routinely referenced during spine surgeries to detect a screw breach, prevent injury of neural elements, and ensure the most biomechanically sound instrumentation construct. To date, similar normative data for cervical lateral mass screws is limited, thus the utility of lateral mass screw testing remains unclear. To address this disparity, in this study the authors describe cumulative lateral mass screw stimulation threshold data in patients undergoing posterior cervical instrumentation with lateral mass screws. These data are correlated with screw placement on postoperative imaging, and a novel correlation is discovered with direct clinical implications.
\end{abstract}

METHODS Using a ball-tip probe, 154 lateral mass screws in 21 patients were electrically tested intraoperatively. In each case, for each screw, the lowest (or threshold) current at which the first polyphasic stimulus-evoked EMG response was reproducibly observed by a neurophysiologist was recorded. All patients underwent postoperative CT. Screw position within the lateral mass was first measured in the axial and sagittal planes for each lateral mass screw using the CT images. Screw placement was also evaluated by 2 independent physicians, blinded to current threshold data, on a binary scale of acceptability. The predictive capacity of screw EMG threshold data was evaluated via multivariable regression analyses and receiver operating characteristic (ROC) analyses. Predictive capacity was examined with respect to screw position within the lateral mass, as well as screw acceptability.

RESULTS Lateral mass screw EMG thresholds did not appear to differ significantly for screws considered "acceptable" versus "unacceptable" according to the radiographic criteria. Accordingly, ROC analysis confirmed that EMG current threshold data were of minimal utility in predicting screw radiographic acceptability. However, EMG threshold was significantly predictive of screw medial distance from the spinal canal. A screw stimulating below $7.5 \mathrm{~mA}$ correctly identified a screw as being within $2 \mathrm{~mm}$ of the spinal canal with $75 \%$ sensitivity and $92 \%$ specificity (positive predictive value $20 \%$, negative predictive value $99.3 \%$ ), independent of its distance relative to other lateral mass landmarks. EMG current threshold was not significantly predictive of screw deviation in the superior or inferior directions, and was inversely predictive of screw deviations in the lateral direction.

CONCLUSIONS In the context of uncertainty regarding the utility of cervical lateral mass EMG current threshold data, this study found that EMG current thresholds correspond significantly, and exclusively, with screw distance from the spinal canal. This association appears independent of other criteria for screw misplacement. As such, the authors recommend that EMG current thresholds be referenced in the case of a suspected medial breach as an effective means to rule out screw placement too medial to the spinal canal.

https://thejns.org/doi/abs/10.3171/2016.8.SPINE16580

KEY WORDS lateral mass; intraoperative; electromyography; medial breach; cervical

ABBREVIATIONS AUC = area under the curve $\mathrm{Cl}=$ confidence interval; $\mathrm{EEG}=$ electroencephalography; $\mathrm{EMG}=$ electromyography; $\mathrm{ROC}=$ receiver operating characteristic; TCMEP = transcranial motor-evoked potential; TIVA = total intravenous anesthesia.

SUBMITTED May 18, 2016. ACCEPTED August 31, 2016.

INCLUDE WHEN CITING Published online December 9, 2016; DOI: 10.3171/2016.8.SPINE16580.

* Mr. Wilson and Dr. Curtis contributed equally to this work. 
$\mathrm{G}$ IVEN the proximity of vital neural and vascular elements to cervical pedicles, ${ }^{11}$ spine surgeons typically target the cervical lateral mass for screw fixation in patients requiring fusion. Despite this operative distinction in the cervical spine, concerns still remain regarding improper screw placement of cervical lateral mass screws. ${ }^{2,9}$ Intraoperative current threshold testing for stimulus-evoked electromyographic responses may serve as a useful tool in this context. Although it remains controversial in the thoracic spine, ${ }^{6,15,17}$ substantial normative electromyographic data for pedicle screw placement in the lumbar spine is routinely referenced intraoperatively to detect screw breach and prevent injury of neural elements. ${ }^{7,13,14,18}$ In the cervical spine, the value of similar data for lateral mass screw placement is unclear; to date only 2 studies have examined the use of intraoperative electromyography (EMG) threshold testing for lateral mass screws, 5,10 and neither has reported definitive recommendations in favor of its utility.

In this study, we describe cumulative lateral screw EMG current threshold data in patients undergoing posterior cervical instrumentation surgery. Furthermore, using postoperative CT to examine screw location accurately, we investigate the association between these EMG current threshold data and screw position within the cervical lateral mass, and discover a novel correlation with direct clinical implications.

\section{Methods}

After obtaining institutional review board approval, 21 consecutive patients undergoing posterior cervical instrumentation at the UC San Diego Medical Center in San Diego, California, or VA Medical Center in La Jolla, California, were enrolled in the study. Patients were excluded if they were over the age of 75 , or if found to carry a known diagnosis of osteoporosis, ankylosing spondylitis, diffuse idiopathic skeletal hyperostosis, traumatic injuries, tumor, or metabolic bone disease. A total of 154 cervical lateral mass screws were placed and successfully tested in the cervical spine between levels C-3 and C-7 (inclusive).

\section{Anesthesia and Neuromonitoring}

Patients underwent intubation using a short-acting paralytic. After positioning the patient prone in rigid fixation, baseline neurophysiological monitoring signals were obtained; train-of-4, spontaneous EMG, stimulus-evoked EMG, transcranial motor-evoked potentials (TcMEPs), somatosensory evoked potentials, and electroencephalography (EEG) recordings were obtained in each case using the Cascade Elite neuromonitoring system (Cadwell Laboratories).

Following positioning, a mixed maintenance anesthetic regimen consisting of propofol and narcotic infusions in combination with a gas anesthetic (sevoflurane, isoflurane, or nitrous oxide) at 0.5 minimum alveolar concentration or less was initiated and adjusted to a depth of anesthesia that correlated with a relative burst-suppression EEG pattern. If adequate TcMEPs and somatosensory evoked potential baseline neurophysiological signals could not be immediately obtained on this mixed anesthetic regimen, a total intravenous anesthesia (TIVA) regimen was implemented. EMG was monitored bilaterally in muscles corresponding to the surgical levels; the trapezius, deltoids, biceps brachia, triceps brachia, thenar, and hypothenar musculature were monitored using 13-mm, 27-gauge subdermal needle electrodes inserted into the belly of each muscle, filtered at high and low cutoff frequencies of $2000 \mathrm{~Hz}$ and $50 \mathrm{~Hz}$, respectively.

Other modalities, including TcMEPs (monitored in the same muscle distribution as the EMG, with the addition of lower limb muscles), somatosensory evoked potentials, trains of 4, and EEG were obtained as previously reported in Allison et al. ${ }^{1}$ Among the baseline signals, a robust trainof-4 response was obtained from the hypothenar muscles via the ulnar nerve prior to lateral mass screw stimulusevoked EMG testing to ensure the effects of the short-acting paralytic administered at intubation had fully worn off.

\section{Screw Placement}

Titanium, polyaxial 3.5-mm-diameter screws were used to instrument the lateral masses of C3-6. After appropriate exposure and palpation, the center of each lateral mass was identified relative to its mediolateral and rostrocaudal borders. Screws were placed 1-2 mm medial to this center position and directed $15^{\circ}$ laterally and $15^{\circ}$ superiorly. All screws were placed by the same attending surgeon or by neurosurgical residents directly supervised by the attending surgeon. The same placement method was used for all lateral mass screws.

\section{Bone and Screw Stimulus-Evoked EMG Testing}

Lateral mass screw stimulus-evoked EMG thresholds were determined with a 50- $\mathrm{ssec}$ constant current monophasic pulse at $3.1 \mathrm{~Hz}$ delivered with a 2.3-mm ball-tip probe (Medtronic), with the anode return placed near midline, rostral to the surgical field. Following screw placement, the probe tip was placed on the screw for stimulation; screws were stimulated just once, at the end of screw placement. For all stimulation procedures, the area surrounding the bone and screw were kept dry with constant suction while the current was increased from $0 \mathrm{~mA}$ to the respective stimulus-evoked EMG current threshold. The EMG current threshold criterion was set as the lowest current at which the first polyphasic compound muscle action potential response was reproducibly demonstrated. Given the unclear utility of EMG current threshold testing for lateral mass screws, no screws were revised intraoperatively upon learning of the EMG current threshold.

\section{Evaluating Screw Directional Deviations}

Following surgery, patients were studied with a CT scan from the occiput to T-2. Using CT images, each screw was assessed for its position within the lateral mass via 4 separate measurements (Fig. 1). These measurements included the distance from the medial-most aspect of each screw to the lateral-most aspect of the spinal canal (termed "medial distance"), the distance from the lateral-most aspect of each screw to the lateral-most aspect of the lateral mass (termed "lateral distance"), the distance from the superiormost aspect of each screw to the superior-most aspect of 
the lateral mass (termed "superior distance"), and the distance from the inferior-most aspect of each screw to the inferior-most aspect of the lateral mass (termed "inferior distance). Each measurement was taken from a point halfway from the screw tip to the base of the polyaxial head of each lateral mass screw in each plane; all medial and lateral measurements were taken in the axial plane, while all inferior and superior measurements were taken in the sagittal plane (Fig. 1). In each case, the shortest distance between landmarks was used as a measurement, as it was believed to reflect the most direct trajectory by which electrical current could travel to the borders of the lateral mass.

\section{Evaluating Screw Acceptability}

To determine placement acceptability for each screw, the CT scans were independently reviewed by a spine surgeon (Dr. Oygar) and a musculoskeletal radiologist (Dr. Chen) blinded to the EMG stimulation data. These reviewers were tasked with grading each screw as either "adequate" (contained entirely within the bone of the lateral mass and of appropriate length) or "inadequate" (inadequate screw angulation, excessive screw length, screw protrusion into either anterior soft tissue or facet joint, and/or a medial or lateral breach). For inadequate screws, the reviewers were also asked to provide an explanation for which specific adequacy criteria were not met. Upon completion of reviewer assessments, screws were categorized as either "acceptable" or "unacceptable" via calculation of a composite score. Adequate screws were given a score of 1 , while inadequate screws were given a score of 0 ; composite scores were then generated for each screw from the sum total of scores from both reviewers. Composite scores equal to 2 were deemed to be in an acceptable position, while composite scores less than 2 were deemed to be in an unacceptable position.

\section{Evaluating Clinical Course}

All patients were followed clinically per standard institutional postoperative care protocols, such that new neurological deficits or complications could be assessed after surgery. All patients were seen and examined by the attending surgeon in the outpatient clinic setting at 2 months after discharge.

\section{Statistical Methods}

The number of patients to be tested was first determined via sample size calculation at study outset, which aimed to discover a 2-mA difference between acceptable and unacceptable screws with $80 \%$ power and $\alpha<0.05$. The results of this calculation revealed that a minimum of 141 screws - which corresponded to approximately 20 patients-would be required to achieve this aim.

To determine the extent of association between screw EMG current threshold and screw directional deviation, a series of linear mixed-effects models, each with a random subject intercept, was generated. Each model set a different directional parameter as the outcome variable (i.e., medial distance, lateral distance, etc.), and set EMG current threshold as the primary predictor. Multivariable analyses for each model included the covariates of age at surgery, cervical side, and cervical level.
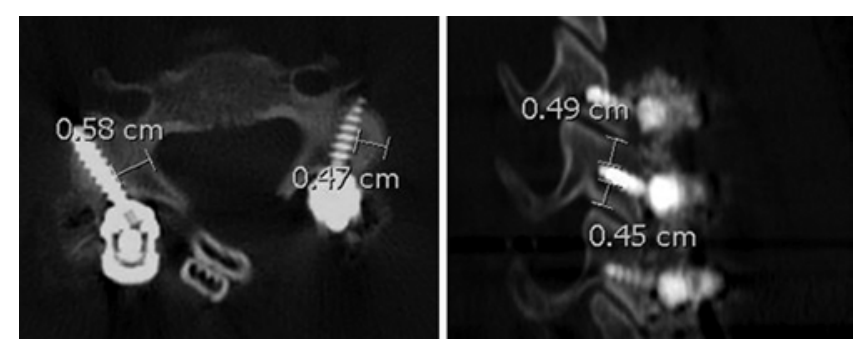

FIG. 1. The 4 measured radiographic distances for each screw. Left: In the axial plane, distances were measured medially from each screw to the vertebral canal (measuring $5.8 \mathrm{~mm}$ in this image), and laterally from each screw to the edge of the lateral mass (measuring $4.7 \mathrm{~mm}$ ). Right: In the sagittal plane, distances were measured superiorly from each screw to the superior edge of the lateral mass $(4.9 \mathrm{~mm})$ and inferiorly from each screw to the inferior edge of the lateral mass $(4.5 \mathrm{~mm})$.

A receiver operating characteristic (ROC) analysis was then performed ${ }^{8}$ to assess the predictive capacity of screw EMG current thresholds for each directional deviation. To perform the ROC analysis, all radiographic directional measures were dichotomized. Screws were described as either "too close" or "safe," according to set distance cutoffs determined by a general consensus among investigators. A screw could be considered too medial $(<2 \mathrm{~mm}$ from the spinal canal), too lateral $(<2 \mathrm{~mm}$ from the edge of the lateral mass), too high ( $<2 \mathrm{~mm}$ of the superior facet), or too low (abutting or within the inferior facet). The criterion for too high was restricted to screws placed $<2$ $\mathrm{mm}$ from the superior facet because no screws were found to abut or protrude into the superior facet. ROC curves for each directional outcome (i.e., too medial, too lateral, etc.) were then examined to determine the EMG current threshold that maximized sensitivity and specificity for identifying that particular directional outcome.

To determine the extent of association between EMG current threshold and screw acceptability as defined by our reviewers, a multivariable logistic regression analysis was performed with screw acceptability as the dependent variable, and screw EMG current threshold as the primary predictor. Covariates for this model were kept consistent with prior linear mixed-effects models and included age at surgery, cervical side, and cervical level. Mean EMG current thresholds for acceptable and unacceptable screws were also compared with an independent samples t-test. Finally, an ROC analysis was performed against the outcome of screw acceptability. Maximal sensitivity and specificity of EMG current thresholds were similarly calculated for the outcome of screw acceptability. Statistical analysis was performed using SPSS (version 23.0, IBM Corp.). All tests were 2 -sided; $p$ values $<0.05$ were considered statistically significant for all final models.

\section{Results}

Twenty-one subjects were enrolled as part of the study protocol, resulting in 154 consecutive lateral mass screws undergoing stimulation. Table 1 illustrates the number of screws placed and tested by spinal level. The mean number of screws stimulated per patient was $7.4 \pm 0.97$; the minimum and maximum number of screws stimulated per patient were 6 and 9, respectively. 
TABLE 1. Number of lateral mass screws stimulated at each cervical level

\begin{tabular}{cc}
\hline Cervical Level & No. of Screws Stimulated \\
\hline C-3 & 30 \\
\hline C-4 & 41 \\
\hline C-5 & 40 \\
\hline C-6 & 39 \\
C-7 & 4 \\
\hline Total & 154 \\
\hline
\end{tabular}

\section{Directional Measurement Data and Predictive Value of EMG Thresholds}

The mean measured distances across all screws are displayed in Table 2. The distributions of screw distances in the medial, lateral, and superior directions were relatively normal, reflecting the fact that no screws appeared to breach medially, laterally, or superiorly according to our independent reviewers. However, there was a significant proportion of screws that were found to have an inferior distance of zero-an observation that correlated strongly with the number of screws deemed to protrude into the corresponding inferior facet by our independent reviewerswhich skewed the inferior distance distribution rightward.

As shown in Table 3, of all 4 radiographic directional parameters assessed, only medial distance was found to have a statistically significant positive association with EMG threshold ( $\mathrm{p}<0.001)$. Specifically, after accounting for patient age, cervical side, and cervical level, screw medial distance from the spinal canal was shown to increase by $0.08 \mathrm{~mm}$ ( $95 \%$ confidence interval [CI] $0.05-0.12 \mathrm{~mm}$ ) for each milliamp increase in screw EMG current threshold (Fig. 2). Lateral distance was also found to be significantly associated with screw EMG current threshold, but this association was in the negative direction $(p=0.002)$, such that an increase in EMG current threshold corresponded to a decreasing distance from the lateral edge of the lateral mass. Superior and inferior distances were not found to have any significant association with screw EMG current threshold.

ROC analyses run for each directional parameter were consistent with the results of our linear mixed-effects models. Screw EMG current threshold was accurate in predicting a medial deviation (i.e., too medial); however, it was inaccurate in this capacity for all other dimensions (i.e., too low, too high, too lateral). The area under the curve (AUC) generated for the medial deviation ROC curve was $0.907(\mathrm{p}=0.006)$, indicating a highly accurate overall testing measure for identifying screws located $<2$ $\mathrm{mm}$ from the spinal canal (Fig. 3). Accordingly, the mean EMG current threshold observed for screws deemed "too medial" (i.e., $<2 \mathrm{~mm}$ from the spinal canal) was significantly lower than for those screws found to be $\geq 2 \mathrm{~mm}$ from the spinal canal (mean difference $7.7 \mathrm{~mA}, 95 \% \mathrm{CI}$ $1.2-14.3, \mathrm{p}=0.02$; Fig. 4).

\section{Sensitivity, Specificity, and Predictive Value}

The current threshold cutoff value that maximized sensitivity and specificity for identifying screws $<2 \mathrm{~mm}$ from
TABLE 2. Directional parameter measurements for all 154 screws

\begin{tabular}{cc}
\hline Directional Parameter & Mean Distance in $\mathrm{mm}(95 \% \mathrm{Cl})$ \\
\hline Superior distance & $4.88(1.40-8.36)$ \\
\hline Inferior distance & $1.26(0.00-3.66)$ \\
\hline Medial distance & $5.00(1.76-8.24)$ \\
\hline Lateral distance & $3.54(0.76-6.32)$ \\
\hline
\end{tabular}

the canal (Fig. 3, arrow) was calculated to be $7.5 \mathrm{~mA}$. Using this cutoff, an EMG current threshold below $7.5 \mathrm{~mA}$ predicted a screw's medial distance to the spinal canal to be less than $2 \mathrm{~mm}$ with $75 \%$ sensitivity and $92 \%$ specificity (positive predictive value $20 \%$, negative predictive value $99.3 \%$ ). An EMG current threshold $\geq 7.5 \mathrm{~mA}$ predicted a screw as being at least $2 \mathrm{~mm}$ from the spinal canal with $99.3 \%$ accuracy.

Table 4 shows a summary of the predictive accuracies of screw EMG current thresholds, ranging from $5.5 \mathrm{~mA}$ to $13.5 \mathrm{~mA}$ for screws deemed to be safe. As expected, increasing amperage corresponded to a higher predictive accuracy in identifying safe screws (i.e., at least $2 \mathrm{~mm}$ from the canal); the minimum threshold value for which predictive accuracy was $100 \%$ was $10.5 \mathrm{~mA}$. Therefore, a screw that stimulated at $\geq 10.5 \mathrm{~mA}$ was shown to predict a medial distance from the spinal canal of at least $2 \mathrm{~mm}$ with $100 \%$ accuracy.

\section{Screw Acceptability and Predictive Value}

Using the scoring methodology described previously (see above), a total of 64 screws (41.5\%) were deemed unacceptable. Of these 64 screws, 21 were considered to be too long (i.e., protruding beyond the lateral mass anteriorly), while 42 were found to protrude into the corresponding inferior facet joint (Fig. 5). No screws were noted to protrude into the superior facet joint, and there were no observed medial or lateral breaches.

As shown in Fig. 6, mean screw EMG current thresholds did not differ significantly between acceptable screws $(14.85 \mathrm{~mA})$ and unacceptable screws $(14.07 \mathrm{~mA} ; \mathrm{p}=0.40)$. Multivariable logistic regression modeling failed to find a significant association between screw EMG current threshold and screw acceptability $(\mathrm{p}=0.47)$. ROC analyses for screw acceptability also failed to generate an AUC of more than 0.55 , indicating an inaccurate test for detecting screws deemed unacceptable by an independent reviewer. Using the available data, the optimum screw EMG current threshold that maximized sensitivity and specificity for screw unacceptability was calculated to be $6 \mathrm{~mA}$. A screw EMG current threshold $<6 \mathrm{~mA}$ predicted an unacceptable screw with $3.1 \%$ sensitivity and $98.9 \%$ specificity; an unacceptable screw on postoperative CT was identified by a screw EMG current threshold of $<6 \mathrm{~mA}$ with $66.6 \%$ accuracy, while an EMG current threshold $\geq 6 \mathrm{~mA}$ identified a screw as acceptable with $58.9 \%$ accuracy.

\section{Postoperative Clinical Course}

Upon reexamination 2 months postoperatively, no study patients were found to have or complain of new neurological signs or symptoms. While a number of patients contin- 
TABLE 3. Linear mixed-effects model (random subject intercept) outputs for each directional deviation parameter

\begin{tabular}{ccccccc}
\hline Directional & \multicolumn{2}{c}{ Univariable } & & \multicolumn{2}{c}{ Multivariable } \\
\cline { 2 - 3 } \cline { 5 - 6 } Parameter $(\mathrm{mm})$ & Estimate Coefficient $(95 \% \mathrm{Cl})$ & & $\mathrm{p}$ Value & & Estimate Coefficient $(95 \% \mathrm{Cl})$ & $\mathrm{p} \mathrm{Value}$ \\
\hline Superior distance & $-0.01(-0.05$ to 0.03$)$ & & 0.67 & & $-0.02(-0.06$ to 0.02$)$ & 0.40 \\
\hline Inferior distance & $-0.02(-0.05$ to 0.01$)$ & & 0.16 & & $-0.02(-0.05$ to 0.01$)$ & 0.25 \\
\hline Medial distance & $\mathbf{0 . 0 9 ( 0 . 0 6 - 0 . 1 3 )}$ & $<0.001$ & & $\mathbf{0 . 0 8 ( 0 . 0 5 - 0 . 1 2 )}$ & $<0.001$ \\
\hline Lateral distance & $-0.05(-0.08$ to -0.02$)$ & & 0.002 & & $-0.05(-0.09$ to -0.02$)$ & 0.002 \\
\hline
\end{tabular}

In each case, screw threshold was set as the main predictor. Multivariable analysis included the covariates of cervical level, patient age at surgery, and cervical side. Boldface type indicates statistically significant positive estimate coefficient.

ued to experience preoperative pain and weakness-albeit improving to varying degrees - there was no observable disparity in persistent signs and/or symptoms between patients with and without at least 1 unacceptable screw, or between patients with and without screws deemed too medial, too lateral, too superior, or too inferior.

\section{Discussion}

Given the general acceptance of normative reference data for pedicle screw EMG current thresholds in the lumbar spine, $, 4,7,14,18$ recent efforts have attempted to generate equivalent data for the cervical spine. To date, available studies have failed to definitively establish firm intraoperative guidelines for interpreting lateral mass screw EMG current threshold data. ${ }^{5,10,12}$ This study, by way of assessing specific directional components of screw location within the lateral mass, presents an alternative means by which lateral mass EMG current threshold data can be interpreted in the intraoperative setting.

The observed disparity in intraoperative guidelines between pedicle screw and lateral mass screw EMG current threshold data can be largely explained by differences in surgical target and technique. In the thoracic and lumbosacral spines, posterior instrumentation procedures target the spinal pedicle, a structure bordered medially by the

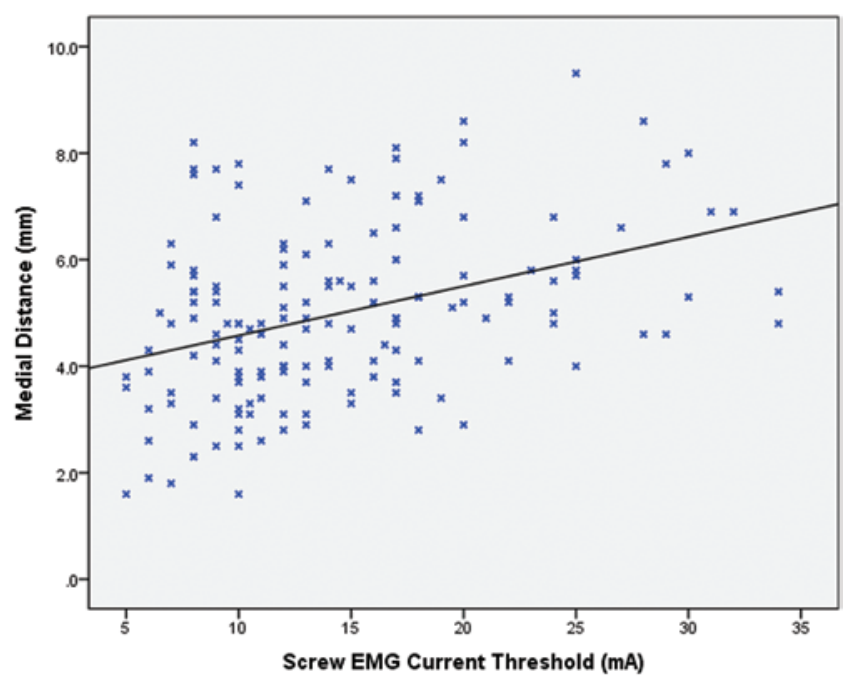

FIG. 2. Screw medial distance plotted as a function of screw EMG current threshold. Only medial distance was shown to correlate positively with EMG current threshold; this correlation was significant $(p<0.001)$. Figure is available in color online only. spinal cord/cauda equina and superiorly and inferiorly by exiting nerve roots. ${ }^{16}$ As such, from the standpoint of intraoperative EMG, deviations in pedicle screw placement in any one of these directions should theoretically correspond to a reduction in EMG current threshold in the appropriate neuromuscular distribution..$^{13}$ For lateral mass screws, a different set of anatomical constraints apply, since screws are directed superiorly and laterally away from the spinal cord. ${ }^{2,19}$ Inaccurate trajectories in the lateral mass, therefore, may not necessarily result in a screw being located closer to exiting nerve roots or to the spinal cord.$^{19}$ As evidenced by our results, a significant number of screws were directed too inferiorly or were too long (i.e., unacceptable), resulting in protrusion into the inferior facet joint or anterior soft tissue, respectively. Our finding that these unacceptable screws were not accurately predicted by EMG current threshold suggests that the radiographic criteria for lateral mass acceptability used for this study - and for other studies to varying degrees ${ }^{5,10}$-are not appropriate for examining the value of lateral mass EMG current threshold data.



FIG. 3. Results of ROC analysis testing the predictive accuracy of EMG current threshold for screws located too medial (i.e., $<2 \mathrm{~mm}$ ) to the spinal canal. The optimum EMG current threshold value for detecting a screw that was too medial (arrow) was calculated to be $7.5 \mathrm{~mA}$. Figure is available in color online only. 


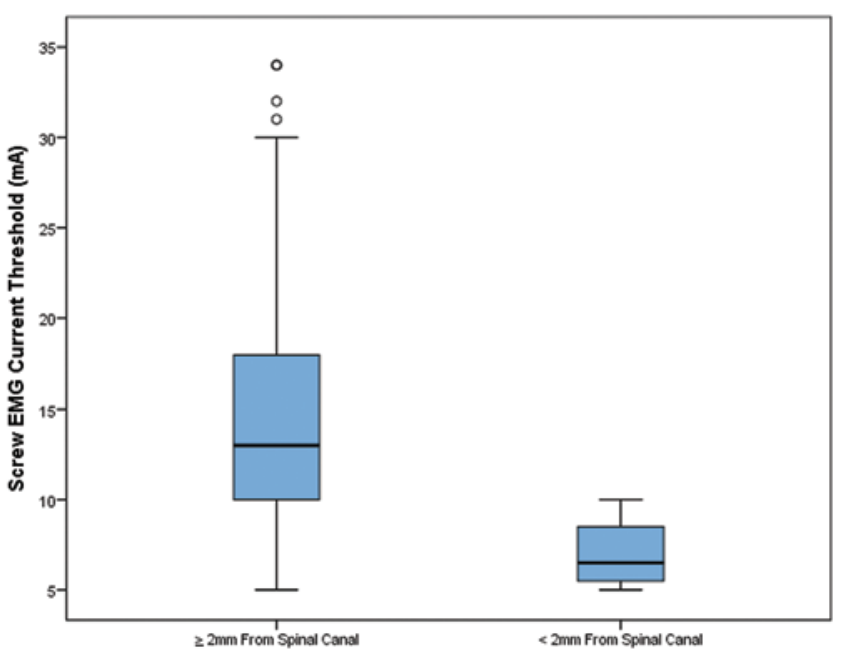

FIG. 4. Bar graph showing EMG current thresholds for screws deemed to be safe (i.e., $\geq 2 \mathrm{~mm}$ from the spinal canal) versus too medial (i.e., $<2 \mathrm{~mm}$ from the spinal canal). A significant difference in EMG current thresholds was observed between groups $(p=0.02)$. Figure is available in color online only.

This study is unique in its assessment of lateral mass EMG current thresholds because it suggests the utility for intraoperative EMG applies mainly to a specific directional deviation within the lateral mass. We contend that in the absence of direct screw contact with a nerve root, EMG current threshold data has significant value in predicting a screw's relative medial distance from the spinal canal. Specifically, our findings suggest that an EMG current threshold value of at least $7.5 \mathrm{~mA}$ predicts with nearly $100 \%$ accuracy that the screw being stimulated is a minimum of $2 \mathrm{~mm}$ away from the spinal canal. Furthermore, because we were unable to find a significant association with either superior of inferior deviation-and found an inverse association with lateral deviation-our findings simplify EMG current threshold interpretation from the perspective of a surgical team, and direct the surgeon and neurophysiologist to primarily consider medial distance when consulting EMG current thresholds for a lateral mass screw.

TABLE 4. Predictive values for a minimum safe distance from the vertebral canal for EMG current thresholds between 5 and $14 \mathrm{~mA}$

\begin{tabular}{cc}
\hline $\begin{array}{c}\text { Minimum Screw EMG } \\
\text { Current Threshold }(\mathrm{mA})\end{array}$ & $\begin{array}{c}\text { Predictive Value for Safe Screw Medial } \\
\text { Distance }(\geq 2 \mathrm{~mm})\end{array}$ \\
\hline 5.5 & 98 \\
\hline 6.5 & 98.6 \\
\hline 7.5 & 99.3 \\
\hline 8.5 & 99.2 \\
\hline 9.5 & 99.1 \\
\hline 10.5 & 100 \\
\hline 11.5 & 100 \\
\hline 12.5 & 100 \\
\hline 13.5 & 100 \\
\hline
\end{tabular}

As amperage increases, the predictive value for a safe medial distance cutoff $(\geq 2 \mathrm{~mm}$ ) also increases. Screws stimulating at $10.5 \mathrm{~mA}$ or higher have a $100 \%$ predictive value of safe medial distance.
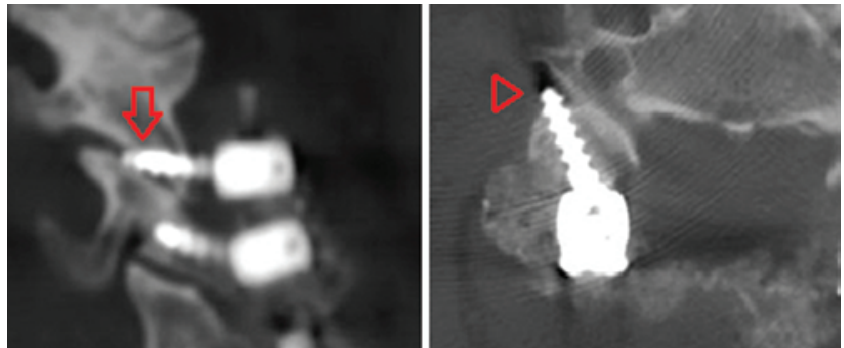

FIG. 5. Examples of unacceptable screws as determined by at least 1 independent reviewer on postoperative CT. Left: A screw found to protrude into the corresponding inferior facet (arrow), which stimulated at $18 \mathrm{~mA}$. Right: A screw found to protrude beyond the lateral mass into anterior soft tissue (arrowhead), which stimulated at $14 \mathrm{~mA}$. Figure is available in color online only.

This is the first such study to report EMG current threshold as a specific predictor of medial proximity to the spinal canal. While previous studies have examined the efficacy of EMG in predicting inaccurate or unacceptable screw placement in more global terms, ${ }^{5,10}$ none has examined screws for specific directional deviations to determine the relative contributions of horizontal and vertical misplacement to EMG current threshold values. In so doing, we have found that EMG current threshold responds exclusively to a screw's horizontal position within the lateral mass, increasing in amperage with increasing medial distance from the spinal canal. This finding is significant as it can inform surgical teams about their risk for medial breach in circumstances in which radiographic and manual investigations fail to confirm a screw as being a safe distance from the spinal canal. Despite the relative rarity of such circumstances, their potential for dramatic neurological implications warrants consideration of this effective and reliable neuromonitoring metric. In such a case, the data in Table 4 can be used to confirm a screw's medial distance as "safe" (i.e., $\geq 2 \mathrm{~mm}$ ) with relative accuracy.

While the authors of this study have since begun rou-

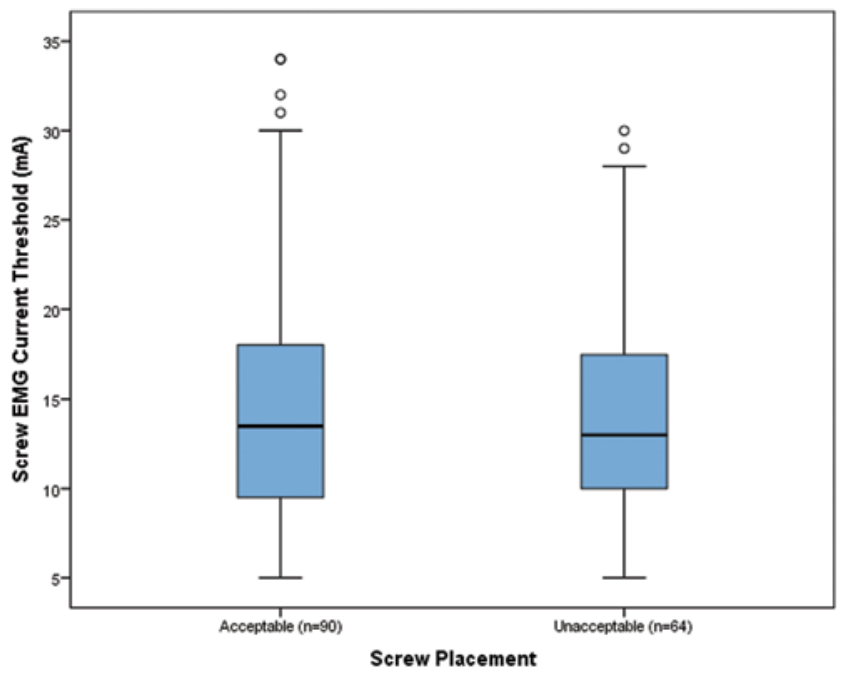

FIG. 6. Bar graph showing EMG current thresholds for screws deemed to be acceptable versus unacceptable. No significant difference in EMG current thresholds was observed between groups $(p=0.40)$. Figure is available in color online only. 
tinely employing EMG testing for lateral mass screws in this context, and would recommend its implementation for all posterior cervical instrumentation procedures, we believe that the decision as to whether to routinely employ EMG testing for lateral mass screws must ultimately fall to an individual surgeon's interpretation of these data, and to his/her personal preference and valuation of confirming a screw's horizontal distance from the spinal canal.

\section{Conclusions}

This study reports a new finding with respect to the utility of lateral mass screw EMG current threshold data. While intraoperative EMG appears to be of minimal value in predicting lateral mass screw acceptability in global terms - a limitation that may be inherent to the anatomy of the lateral mass-it can serve as a powerful predictor of screw medial distance from the spinal canal. As such, it should be consulted as an additional reference during intraoperative scenarios in which medial breach is suspected.

\section{Acknowledgments}

The project described was partially supported by grant no. 1TL1TR001443 from the NIH. The content is solely the responsibility of the authors and does not necessarily represent the official views of the NIH. We would like to acknowledge the following individuals for their contribution to the study: Jayson Sack, MD, MS; Matthew MacDougall, MD; Pawel Jankowski, MD; Reid Hoshide, MD, MPH; David Santiago-Dieppa, MD; Jeffrey Steinberg, MD; Joel Martin, MD; Robert Rennert, MD; and Mihir Gupta, MD.

\section{References}

1. Allison DW, Gertsch JH, Mahan MA, Sheean GL, Brown JM: Anesthesia considerations for monitoring TCMEPs in adults diagnosed with poliomyelitis as children: a case report. Neurodiagn J 54:28-35, 2014

2. An HS, Gordin R, Renner K: Anatomic considerations for plate-screw fixation of the cervical spine. Spine (Phila Pa 1976) 16 (10 Suppl):S548-S551, 1991

3. Bose B, Wierzbowski LR, Sestokas AK: Neurophysiologic monitoring of spinal nerve root function during instrumented posterior lumbar spine surgery. Spine (Phila Pa 1976) 27:1444-1450, 2002

4. Calancie B, Madsen P, Lebwohl N: Stimulus-evoked EMG monitoring during transpedicular lumbosacral spine instrumentation. Initial clinical results. Spine (Phila Pa 1976) 19:2780-2786, 1994

5. Djurasovic M, Dimar JR II, Glassman SD, Edmonds HL, Carreon LY: A prospective analysis of intraoperative electromyographic monitoring of posterior cervical screw fixation. J Spinal Disord Tech 18:515-518, 2005

6. Donohue ML, Murtagh-Schaffer C, Basta J, Moquin RR, Bashir A, Calancie B: Pulse-train stimulation for detecting medial malpositioning of thoracic pedicle screws. Spine (Phila Pa 1976) 33:E378-E385, 2008

7. Glassman SD, Dimar JR, Puno RM, Johnson JR, Shields CB, Linden RD: A prospective analysis of intraoperative electromyographic monitoring of pedicle screw placement with computed tomographic scan confirmation. Spine (Phila Pa 1976) 20:1375-1379, 1995

8. Greiner M, Pfeiffer D, Smith RD: Principles and practical application of the receiver-operating characteristic analysis for diagnostic tests. Prev Vet Med 45:23-41, 2000
9. Heller JG, Silcox DH III, Sutterlin CE III: Complications of posterior cervical plating. Spine (Phila Pa 1976) 20:24422448, 1995

10. Holdefer RN, Heffez DS, Cohen BA: Utility of evoked EMG monitoring to improve bone screw placements in the cervical spine. J Spinal Disord Tech 26:E163-E169, 2013

11. Jones EL, Heller JG, Silcox DH, Hutton WC: Cervical pedicle screws versus lateral mass screws. Anatomic feasibility and biomechanical comparison. Spine (Phila Pa 1976) 22:977-982, 1997

12. Kelleher MO, Tan G, Sarjeant R, Fehlings MG: Predictive value of intraoperative neurophysiological monitoring during cervical spine surgery: a prospective analysis of 1055 consecutive patients. J Neurosurg Spine 8:215-221, 2008

13. Parker SL, Amin AG, Farber SH, McGirt MJ, Sciubba DM, Wolinsky JP, et al: Ability of electromyographic monitoring to determine the presence of malpositioned pedicle screws in the lumbosacral spine: analysis of 2450 consecutively placed screws. J Neurosurg Spine 15:130-135, 2011

14. Raynor BL, Lenke LG, Bridwell KH, Taylor BA, Padberg AM: Correlation between low triggered electromyographic thresholds and lumbar pedicle screw malposition analysis of 4857 screws. Spine (Phila Pa 1976) 32:2673-2678, 2007

15. Raynor BL, Lenke LG, Kim Y, Hanson DS, Wilson-Holden TJ, Bridwell KH, et al: Can triggered electromyograph thresholds predict safe thoracic pedicle screw placement? Spine (Phila Pa 1976) 27:2030-2035, 2002

16. Roy-Camille R, Saillant G, Mazel C: Internal fixation of the lumbar spine with pedicle screw plating. Clin Orthop Relat $\operatorname{Res}(\mathbf{2 0 3}): 7-17,1986$

17. Samdani AF, Tantorski M, Cahill PJ, Ranade A, Koch S, Clements DH, et al: Triggered electromyography for placement of thoracic pedicle screws: is it reliable? Eur Spine J 20:869-874, 2011

18. Shi YB, Binette M, Martin WH, Pearson JM, Hart RA: Electrical stimulation for intraoperative evaluation of thoracic pedicle screw placement. Spine (Phila Pa 1976) 28:595-601, 2003

19. Xu R, Haman SP, Ebraheim NA, Yeasting RA: The anatomic relation of lateral mass screws to the spinal nerves. A comparison of the Magerl, Anderson, and An techniques. Spine (Phila Pa 1976) 24:2057-2061, 1999

\section{Disclosures}

The authors report no conflict of interest concerning the materials or methods used in this study or the findings specified in this paper.

\section{Author Contributions}

Conception and design: Ciacci, Wilson, Curtis, Gabel. Acquisition of data: Ciacci, Wilson, Oygar, Chen, Allison. Analysis and interpretation of data: Ciacci, Wilson, Curtis, Hirshman, Allison. Drafting the article: Ciacci, Wilson, Curtis. Critically revising the article: Ciacci, Wilson, Curtis, Gabel, Vaida, Allison. Reviewed submitted version of manuscript: all authors. Approved the final version of the manuscript on behalf of all authors: Ciacci. Statistical analysis: Ciacci, Wilson, Hirshman, Vaida. Administrative/ technical/material support: Ciacci, Wilson. Study supervision: Ciacci, Curtis.

\section{Correspondence}

Joseph D. Ciacci, Department of Neurosurgery, University of California, San Diego, 200 West Arbor Dr. \#8893, San Diego, CA 92103. email: jciacci@ucsd.edu. 